\title{
Das JfB gehört zu den meistgelesenen wirtschaftswissenschaftlichen Zeitschriften
}

\section{Barbara Sporn}

Online: 22. Oktober 2008

(C) Wirtschaftsuniversität Wien, Austria 2008

Das Journal für Betriebswirtschaft (JfB) wurde 1951 an der damaligen Wiener Hochschule für Welthandel, der heutigen Wirtschaftsuniversität Wien gegründet. Es gehört damit international zu den traditionsreichsten und ältesten wissenschaftlichen Zeitschriften im Bereich der Betriebswirtschaftslehre und wird bis heute von der Wirtschaftsuniversität Wien getragen.

Im Zuge der neuen Forschungsstrategie der WU, die eine weitere Verbesserung der Wettbewerbsposition durch verstärkte Profilbildung und noch deutlichere internationale Präsenz vorsieht, wurde im Jahr 2003 auch das JfB grundlegend neu positioniert. Elemente dieser Neuausrichtung waren unter anderem der Verlagswechsel zu Springer, erweiterte Beiräte und wesentliche Veränderungen in den internen Prozessen, insbesondere im Begutachtungsprozess. Dabei erfolgte eine Orientierung an höchsten internationalen Standards. Die wichtigste Änderung war aber die inhaltliche Neupositionierung. Seit 2005 werden im JfB ausschließlich innovative Übersichtsartikel publiziert, die den State of the Art der internationalen Forschung zu abgegrenzten und relevanten Forschungsfeldern strukturiert bewerten, sowie neue Richtungen aufzeigen. Damit wird eine wichtige Lücke in der zunehmend unübersichtlich werdenden Forschungslandschaft geschlossen.

Heute, fünf Jahre nach der Entscheidung der WU zur Neupositionierung des JfB und drei Jahre nach dem tatsächlichen Erscheinen des ersten Heftes kann eine Bilanz des Erreichten gezogen werden.

Die vor wenigen Wochen vom Verband der Hochschullehrer für Betriebswirtschaft veröffentlichten Ergebnisse einer Befragung, an der sich insgesamt 1010 Wissenschaftler/innen im deutschsprachigen Raum beteiligten, ergab das erfreuliche Ergebnis, dass das JfB unter allen 682 erfassten wissenschaftlichen Zeitschriften im Be-

B. Sporn (

Forschung, International und External Relations, Wirtschafstuniversität Wien, Nordbergstraße 15, 1090 Wien, Österreich

E-Mail: barbara.sporn@wu-wien.ac.at 
reich BWL die achtgrößte Leser/inn/en/basis hat. Selbst international führende Spitzenzeitschriften, wie das Academy of Management Journal, Management Science oder Administrative Science Quarterly werden von wesentlich weniger Betriebswirt/inn/en in Österreich, Deutschland und der Schweiz gelesen. Damit leistet das JfB einen außerordentlich wichtigen Beitrag zur Etablierung der Wirtschaftsuniversität Wien als führende Forschungseinrichtung im deutschsprachigen Raum. Deutlich wird dadurch aber auch, dass Autorinnen und Autoren mit einer Publikation im JfB tatsächlich die Scientific Community erreichen.

Gegenüber dem ,,alten“ VHB Zeitschriftenranking, das 2003, kurz vor der Neupositionierung des JfB, publiziert wurde, hat sich das JfB auch hinsichtlich seiner wissenschaftlichen Reputation im Verhältnis zu seinen direkten Wettbewerbern, den allgemein-betriebswirtschaftlich ausgerichteten deutschsprachigen Zeitschriften, äußerst positiv entwickelt. Das JfB hat zu den oft als die ,großen Drei“ bezeichneten traditionellen Marktführern im deutschsprachigen Raum (Zeitschrift für Betriebswirtschaft, Zeitschrift für betriebswirtschaftliche Forschung und Die Betriebswirtschaft) aufgeschlossen und ist nun erstmals hinsichtlich seiner wissenschaftlichen Reputation die Nummer Vier in diesem Marktsegment.

Die Erhebungen des Verbandes der Hochschullehrer für Betriebswirtschaft aus den Jahren 2003 und 2008 verdeutlichen aber auch den Trend, dass es Zeitschriften des deutschsprachigen Raumes zunehmend schwerer haben, sich unter den internationalen Zeitschriften zu behaupten. Unter den ABWL-Zeitschriften des deutschsprachigen Raumes hat sich einzig das JfB gegen den ansonsten durchgängigen Trend einer Abnahme des Ansehens entwickelt. Damit wird deutlich, dass es im dynamischen Wettbewerb der wissenschaftlichen Zeitschriften darauf ankommt, sich ständig weiter zu entwickeln und neuen Herausforderungen zu begegnen. Die Wirtschaftsuniversität Wien wird als Trägerin des JfB die Herausgeber weiterhin tatkräftig unterstützen und ich bin sicher, dass alle Herausforderungen wie in der Vergangenheit mit unternehmerischer Initiative gemeistert werden und sich das JfB auch in Zukunft weiter sehr positiv entwickeln wird. Mein herzlicher Dank gilt den beiden Herausgebern, Nikolaus Franke und Gerhard Speckbacher, für ihre unermüdliche Arbeit im Dienst der Qualität des JfB.

Wien, Oktober 2008

Barbara Sporn 